\title{
The Method of SAR RFI Suppression Based on Compressed Sensing
}

\author{
Kong Shuya ${ }^{1, a}$, Ye Wei ${ }^{1}$, Lao Guochao ${ }^{1}$,Zhang Weikun ${ }^{1}$ \\ ${ }^{1}$ The Academy of Equipment, Beijing 101416,China \\ a494926081@qq.com
}

\begin{abstract}
Keywords: Compress Sensing, Synthetic Aperture Radar, RFI Suppression, Minimum Description Length
\end{abstract}

\begin{abstract}
To the synthetic aperture radar (SAR) imaging system which using Compressed Sensing (CS) technique,radio frequency interference (RFI) would undermine the priori sparse condition and cause deteriorationof image quality. In this paper, under the condition of the sparse target scene, it builds the target echo signal and RFI redundant dictionary, according to RFI sparse features on their redundant dictionary, greedy algorithm was used in conjunction with the minimum description length (MDL) criterion to estimate the sparsenessof RFI, and SAR imaging RFI suppression algorithms on the compressed domain was designed based on this basis. Simulation results show that in the larger JSR conditions the algorithmcan effectively suppress SAR radio interference and achieve better imaging results.
\end{abstract}

\section{I .INTRODUCTION}

Synthetic Aperture Radar (SAR) are not affected by the weather and time limitations, in military and civil fields it has been widely used, but large amount of data caused by large bandwidth brings great burden to store and transport system[1]. Compressive Sensing (CS) technology can achieve good imaging results by using a small amount of data based on the signal sparse sampling and reconstruction precision; but due to the intrinsic characteristics of CS theory, when the SAR echo signal exists strongRFI, the SAR image quality will be serious decline[2]. Therefore, it is of great significance for the SAR imaging to study how to detect and inhibition the RFI.

Existing RFI suppression methods can be divided into parametric method and nonparametric method[3]. The main idea of parametric method is modeled on the RFI, considering the RFI as a model with many constant amplitude single frequency signal superimposed or random process in compliance with the AR model, using minimum mean square error (LMS) or maximum likelihood (Maximun likelihood, ML) criterion to estimate the signal model parameters, and then use the estimate - subtraction structure to filter the obtained RFI estimated from the raw data[4]; however, in the case of highly intensive RFI modeling are more complex, it will produce the model parameter errors and computationally intensive problems. Non-parametric methods are usually detecting the interference and signal based on signal characteristics RFI with the use of spectrum estimation method, then suppressing the RFI on the time-frequency domain using the filtering method. It mainly includes the frequency domain filtering method, subspace filtering method and image subtraction method[5]. Frequency domain notch method suppresses the interference by way of making the position of RFI the frequency domain zero;however, the signal will be filtered out at the same position in the frequency domain while suppressinginterference,resulting in the loss of signal energy[6]. Subspace filtering method projects the interference and signal, then filtering the corresponding subspace of interference out, this method can suppress the steady RFI better, butfor distance-changingRFI it will have a greater signal loss when suppressing interference[7]. Subtraction imaging through a sit channel to get RFI without signal filtered it out in the image data directly; however, this method requires a separate sit channel[7].

In summary, although the existing method can suppress RFI, but there are some problems of energy loss, intensive computation and high complexity modeling. Within the spectrum of SAR, RFI components in the frequency domain has obvious sparse features, the number of principal 
components (the sparseness of RFI) of RFI components can be accessed by OMP algorithm combined with minimal description length criterion (MDL). Because RFI has high emission power, one-way communication and other characteristics, so the energy of RFI components in the frequency domain is higher than SAR echo signal.Based on this, each echo received by the SAR, firstly, uses the OMP algorithm to reconstruct the interference coefficient and set it to zero, then uses the SAR echo signal dictionary to carry on the compressed sensing image.

\section{II.CS BASIC THEORY}

For a $\mathrm{N}$ dimensional signal $x\left(x \in \mathbf{R}^{N \times 1}\right)$ whose projection coefficient on the $N \times N$ basis matrix $\boldsymbol{\Psi}=\left\{\psi_{1}, \psi_{2}, \cdots, \psi_{i}, \cdots, \psi_{N}\right\}\left(\psi_{i}\right.$ is a $\mathrm{N}$ dimensional vector) were only contain $K(K<<)$ non-zero value, then we know the signal $x$ could be sparse on dictionary $\boldsymbol{\Psi}$, The sparse coefficient $\boldsymbol{\alpha}$ can be obtained by the inner product $x$ and $\boldsymbol{\Psi}$, that is $\alpha_{i}=<x, \psi_{i}>$.

The signal $x$ can be expressed as

$$
x=\sum_{i=1}^{N} \alpha_{i} \psi_{i} \quad \text { or } \quad \mathbf{x}=\Psi \boldsymbol{\alpha}
$$

The assumption that $\boldsymbol{\Phi}$ is a $M \times N$ dimensional measurement matrix, then we got a $M \times 1$ dimension signal $y$ after linear measurement, that is

$$
\mathbf{y}=\Phi \mathbf{x}=\Phi \Psi \alpha=\Theta \alpha
$$

Where $\boldsymbol{\Theta}=\boldsymbol{\Phi} \boldsymbol{\Psi}$ is a $M \times N$ perception matrix.

If the perceptual matrix $\Theta$ satisfied the restricted isometry property (RIP)[8], that is, $1-\varepsilon \leq\|\boldsymbol{\Theta} x\|_{2}^{2} /\|x\|_{2}^{2} \leq 1+\varepsilon, \varepsilon>0$, then the signal can be reconstructed by solving the $l_{0}$ norm problem. That is,

$$
\hat{\alpha}=\arg \min \|\alpha\|_{0} \quad \text { s.t. } \quad \mathbf{y}=\boldsymbol{\Theta \alpha} \quad \text { (3) }
$$

In the formula, $\|\cdot\|_{0}$ is the $l_{0}$ norm,that is the number of nonzero elements in the vector $\boldsymbol{\alpha}$.

The commonly used reconstruction algorithms are greedy algorithm and convex optimization algorithm. This paper uses the improved OMP algorithm to reconstruct the signal which considered the complexity and reconstruction precision of the algorithm.

\section{III.SAR RFI SUPPRESSIN METHOD BASED ON COMPRESSIVE SENSING}

\section{A. SAR Target Echo and RFI Sparse Dictionary Construction}

a)The model and sparse representation of SAR target echo signal

If $s_{0}(t)=\operatorname{rect}\left[\left(t / T_{r}\right) / T_{r}\right] \cdot \exp \left(j \pi k_{r} t^{2}\right)$, then the SAR baseband echo signal is

$$
\left.s_{r}(\eta, t)=\sum_{m=1}^{M}\left\{\alpha_{m}(\eta) s_{0}\left[t-2 r_{m}(\eta) / c\right)\right]\right\}+n_{s}(t)
$$

In the formula, $\alpha_{m}(\eta)=T_{r} \sigma_{m} \exp \left[-j 4 \pi f_{0} r_{m}(\eta) / c\right] \cdot \sigma_{m}$ is the backscattering coefficient of the first $\mathrm{m}$ scattering point, $f_{0}$ is the center frequency of the linear frequency modulation signal, $r_{m}(\eta)$ is the instantaneous distance from the radar platform to the m scattering center at $\eta$ pulse emission point, $T_{r}$ is the transmission of the linear FM signal pulse width, $c$ is the speed of light, $n_{s}(t)$ for the radar beam radiation area of the weak scattering center echo formation of the equivalent noise.

Assuming that the resolution range of the radar in the observation scene is $\Delta r$, the minimum distance of the scene is $r_{0}$, the maximum distance is $r_{1}$, then the distance to the scene can be divided into $q=\left(r_{1}-r_{0}\right) / \Delta r$ parts, and the scattering center of the target in the scene range unit can be expressed by one dimension vector $\boldsymbol{\alpha}_{s}$, that is,

$$
\boldsymbol{\alpha}_{s}^{T}=\left[\begin{array}{llll}
\alpha_{1} & \alpha_{2} \cdots \alpha_{q-1} & \alpha_{q}
\end{array}\right]_{1 \times q}
$$

In the formula, $r_{q}=r_{0}+q \Delta r, q \in[0, \mathrm{q}-1]$ is the instantaneous distance between the $q$ unit and the 
radar platform. Assuming that there are only $m$ targets in the observation scene, the number of non-zero elements in $\boldsymbol{\alpha}_{s}$ is much smaller than that of the number of the distance units, that is, the echo signal of the target in the scene is sparse.

We carry on the time delay of the signal $s_{0}(t)$, then we can build the echo signal sparse dictionary.

b) The model and sparse representation of RFI signal

RFI is a narrowband Gauss process which probability distribution follows normal distribution. The model of RFI is:

$$
J(t)=U_{n}(t) \cos \left[\omega_{j} t+\varphi(t)\right]
$$

The envelope function $U_{n}(t)$ obeys the Rayleigh distribution, phase function $\varphi(t)$ obeys $[0,2 \pi]$ uniform distribution and is relatively independent with $U_{n}(t)$, carrier frequency $\omega_{j}$ is a constant and is much larger than the spectral width of $J(t)$.The sparse representation of RFI with Fourier basis is:

$$
\mathbf{s}_{\mathbf{j}}=\boldsymbol{\Psi}_{D F T} \boldsymbol{\alpha}_{j}
$$

In the formula, $\mathbf{s}_{\mathbf{j}}$ is column vector representation, $\boldsymbol{\alpha}_{j}$ is a $N \times 1$ projection coefficient vector based on $\boldsymbol{\Psi}_{D F T}$.

c)Sparseness estimation of RFI

OMP algorithm needs to input the number of iterations of the algorithm, that is, the sparseness $k_{j}$ of the RFI signal as a priori conditions, but the RFI component is usually unknown, therefore, need to estimate it.The possibility of receiving a change of RFI in the synthetic aperture time of SAR is very small, so we can estimate the value of $k_{j}$ by take the compression sampling data of the first $R$ echo pulse. The statistical average can be as the sparseness of the reconstruction of each echo. In order to reduce the estimation error, a conservative sparseness $K_{R}$ is selected as the number of iterations of the OMP algorithm firstly. For example, the length of the signal is equal to $N$, make $K_{R}=N / 4$, that is, the spectral range of RFIrepresenting1/4 of the target echo signal. In this way, use the first $R$ pulse compressed data $r=1,2, \cdots R$,canreconstruct thefrequency-domainsample data $\hat{S_{j}^{(r)}}=\left[\begin{array}{lllll}\hat{S_{1}^{(r)}} & \hat{S_{2}^{(r)}} & \cdots & \hat{S_{N}^{(r)}}\end{array}\right]^{T}$ bythe OMPalgorithm, the non-zero elements is frequency-domain sampling of the first $K_{R}$ large modulus. Statistical average modulus value foreach element can get $\bar{S}_{j}=\left[\begin{array}{llll}\bar{S}_{1} & \bar{S}_{2} & \cdots & \bar{S}_{N}\end{array}\right]^{T}$, its element value is

$$
\bar{S}_{i}=\frac{1}{R} \sum_{r=1}^{R}\left|\hat{S_{i}^{(r)}}\right| \quad i=1,2, \cdots, \mathrm{N}
$$

Due to the impact of target echo data, Non - zero element of $\bar{S}_{j}$ is not $K_{R}$, but the number of its principal components (the number of elements in the modulus value obviously large) can be used as the estimated value of $k_{j}$, The number of principal components can be estimated using MDL criteria[9]. Assuming the number of non-zero elements is $L$, sort the value of these elements $\lambda_{1} \in\left\{\begin{array}{cccc}\bar{S}_{1} & \bar{S}_{2} & \cdots & S_{N}\end{array}\right\}, l=1,2, \cdots, L$ from large to small ,that is, $\lambda_{1} \geq \lambda_{2} \geq \cdots \lambda_{L}$. Sorted data is calculated as following formula:

$$
M D L(l)=2 L(L-l) \rho(l)+\frac{1}{2} l(2 L-l) \lg (2 L) \quad l=0,1,2, \cdots, L-1
$$

In the formula,

$$
\rho(l)=\lg \left(\sum_{i=l+1}^{L} \lambda_{i}\right)-\lg (L-1)-\frac{1}{L-l} \sum_{i=l+1}^{L} \lg \lambda_{i}
$$


The minimum value of $M D L(l)$ is the estimation of principal component number:

$$
k_{j}=\min _{l} M D L(l)
$$

If $\mathrm{K} \neq 0$, There is RFI exist in the compressed sample data.

\section{B. Theory Analysis of Algorithm}

The representation of SAR echo data with RFI is:

$$
\mathbf{X}=\boldsymbol{\Psi}_{s} \boldsymbol{\alpha}_{s}+\Psi_{D F T} \boldsymbol{\alpha}_{j}
$$

the representation of $\mathbf{X}$ by CS observation is:

$$
\begin{aligned}
\mathbf{Y} & =\boldsymbol{\Phi} s_{r}(\eta, t)+\boldsymbol{\Phi} \mathbf{s}_{\mathbf{j}} \\
& =\boldsymbol{\Phi} \boldsymbol{\Psi}_{s} \boldsymbol{\alpha}_{s}+\boldsymbol{\Phi} \boldsymbol{\Psi}_{D F T} \boldsymbol{\alpha}_{j} \\
& =\boldsymbol{\Phi}\left[\boldsymbol{\Psi}_{s}, \boldsymbol{\Psi}_{D F T}\right]\left[\begin{array}{l}
\boldsymbol{\alpha}_{s} \\
\boldsymbol{\alpha}_{j}
\end{array}\right]
\end{aligned}
$$

In the formula, Observation matrix $\boldsymbol{\Phi}$ is a $M \times N(M<N)$ dimensional Gauss matrix.

RFI have Characteristic of high power and narrow bandwidth, in the spectral range of SAR show the peak,and SAR echo has a relatively flat power spectrum, the energy of the RFI component is higher than that of the SAR echo signal. Therefore, mixed signal with interference have to match Fourier atoms first to reconstruct the interference coefficient.

Previous studies have shown that Fourier basis is not related to the target echo signal dictionary.Therefore, firstly, based on the Fourier basis $\boldsymbol{\Psi}_{D F T}$, to reconstruct RFI by using the OMP algorithm. Put the compressed data of each pulse $y$, perceptual matrix $\Theta$ and sparseness $k_{j}$ into the OMP algorithm, get $k_{j}$ RFI component reconstruction results which corresponding $\mathbf{s}_{\mathbf{j}}$, recording there frequency-domain location $\left\{p_{1,} p_{2}, \cdots, p_{k}\right\}$, then set the reconstruction coefficient to zero of the corresponding position.

For received each echo, the data after the RFI suppressed is reconstructed with the dictionary $\Psi_{s}$ of the target echo signal, can get the range image which after the interference suppression, and the final imagecould obtained by using the traditional matched filter in azimuth direction.

\section{Algorithmic process}

Step1>Initializationresiduals $r_{1}=y$, coefficient vector hat $y=\varnothing$, incrementmatrix Aug $t=[]$, loopiterations number $t=1$;

Step2> Execution OMPalgorithm, ergodicperceptionmatrix $\Psi_{D F T}$ of RFIsignals, until $t=k_{j}$, output residuals $r_{2}$, update the coefficient vector matrix hat $y$ and incrementmatrix Aug $t$;

Step3 $>$ Reset $t=1$, order residuals $r_{2}=r_{1}$;

Step4> Execution OMP algorithm again, ergodic perception matrix $\boldsymbol{\Psi}_{s}$ of target echo signal, Until $t=k_{s}$, Output reconstructioncoefficient vector $h a t_{-} y$ and the matchedAtomic matrix Aug $t$;

Step5>For each echo, put the reconstructed interference coefficient tozero according to Step1 Step4, get the one-dimensional distance.

Step6>To get the final imaging resultsby using azimuthmatching filtering.

\section{SIMULATION EXPERIMENT}

It is assumed that there are five strong scattering points in the imaging scene and the size of the imaging scene is $150 \mathrm{~m} \times 100 \mathrm{~m}$, that is, the sparse degree of the target is 5 .

In the simulation, the center frequency of the side looking airborne SAR is $3 \mathrm{GHz}$, and its specific parameters are shown in table 1 . There are $N=512$ sampling points in the range direction, add the RFI signal with a bandwidth of $5 M$ at the frequency of $2.985 \mathrm{GHz}$. By using the method provided in this paper, the number of principal components of RFI is estimated to be 33 (that is, the sparseness of RFI is 33). Simulation defined compression ratio $M$ is equal to actual sampling points 
than the Nyquist sampling points, set the compression ratio $M$ is equal to 2, namely the down sampled with 256 sampling point.

Table 1 The side looking SAR parameters

\begin{tabular}{lc}
\hline carrier frequency & $3 \mathrm{GHz}$ \\
pulse width & $1.5 \mu \mathrm{s}$ \\
bandwidth & $150 \mathrm{MHz}$ \\
sampling frequency & $180 \mathrm{MHz}$ \\
pulse repetition frequency & $188 \mathrm{MHz}$ \\
Imaging time & $1.4 \mathrm{~s}$ \\
\hline
\end{tabular}

When $J S R=35 \mathrm{~dB}$, the sparseness of the scene is severely damaged. SAR imaging algorithm based on CS has been unable to imaging, as shown in Figure 1:
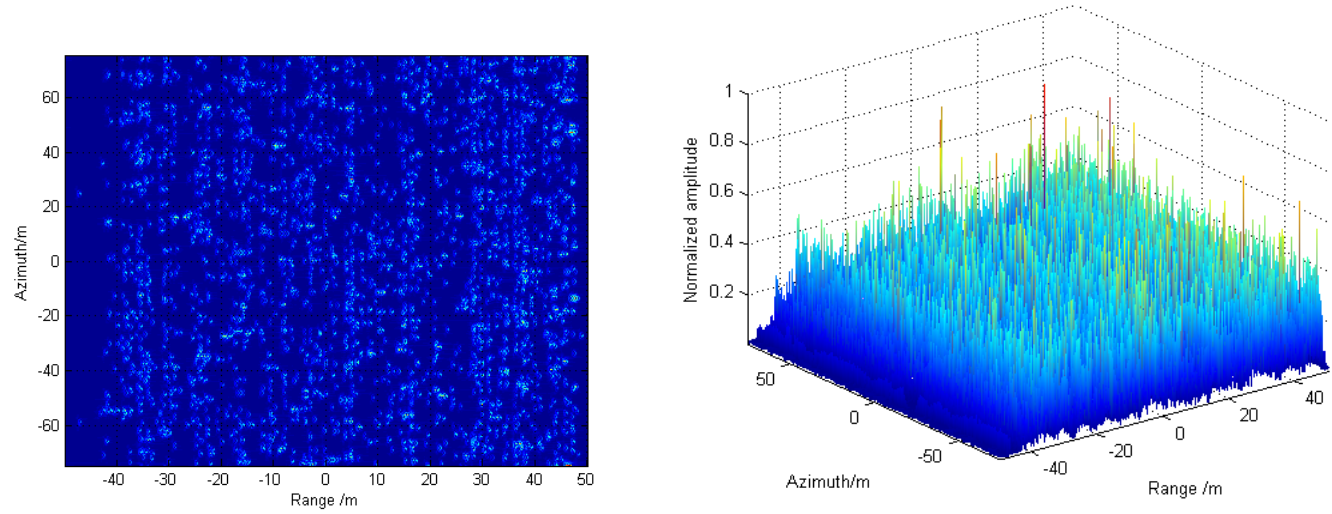

a) When $J S R=35 d B$, imaging results after compressionb) When $J S R=35 d B$, Two dimensional compression after imaging results

Figure 1 When $J S R=35 d B$, SAR imaging results based on CS

Under the condition of $J S R=35 \mathrm{~dB}$, the method provided in this paper can effectively suppress the interference and achieve a better imaging effect, as shown in Figure 2:
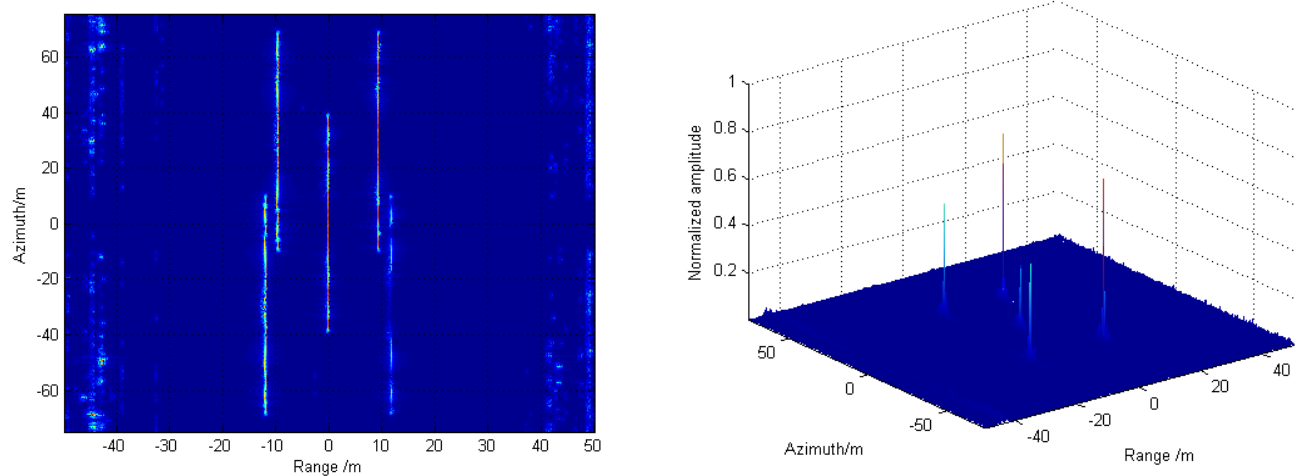

a) When $J S R=35 d B$, imaging results after compressionb) When $J S R=35 d B$, Two dimensional compression after imaging results

Figure 2 When $J S R=35 d B$, Results of suppress the interference of SAR imaging results based on CS Under different JSR conditions, Recover PSLR and ISLR of target as shown in Table 2:

Table 2 Image quality index of point target

\begin{tabular}{|c|c|c|c|c|}
\hline \multirow{2}{*}{ JSR (dB) } & \multicolumn{2}{|c|}{ PSLR(dB) } & \multicolumn{2}{c|}{ ISLR(dB) } \\
\cline { 2 - 5 } & $\begin{array}{c}\text { Range } \\
\text { Direction }\end{array}$ & Azimuth & $\begin{array}{c}\text { Range } \\
\text { Direction }\end{array}$ & Azimuth \\
\hline No interference & 17.768 & 13.155 & -9.841 & -5.689 \\
\hline 15 & 16.846 & 12.174 & -8.955 & -5.716 \\
\hline 25 & 16.633 & 8.542 & -8.632 & -4.694 \\
\hline 35 & 16.574 & 8.109 & -8.792 & -4.091 \\
\hline
\end{tabular}


As can be seen from the table 2, under different JSR conditions, after interference suppression processing, PSLR and ISLR of point target are very similar to the echo signal without interference. This also shows the effectiveness of the method.

\section{CONCLUSIONS}

This paper analyzed the effects of the RFI on compressive sensing SAR imaging, on the basis of estimating the RFI sparseness with MDL criterion, it uses OMP reconstruction algorithm to design an RFI suppression method. According to the differences between RFI signals and SAR target echo signal on the sparse domain, the method constructs a dictionary to reconstruct them, and achieves the purposes of RFI suppression. This method does not require RFI sparseness as a priori information, it has some practical value in the SAR signal processing.

\section{REFERENCE}

[1] CUMMING I G, WONG F.Digital processing of syntheticaperture radar data: algorithms and implementation [M]. Norwood: Artech House.2005: 1-35.

[2] Lin Y, Huifang Z, Jin F, et al. Detection and suppression of narrow band RFI for synthetic aperture radar imaging [J].Chinese Journal of Aeronautics. 2015(04): 1189-1198.

[3] WANG Yanping, PENG Hailiang, WU Yirong.etal.Summaryof narrow band interference suppression in syntheticaperture radar[J]. Modern Defence Technology, 2003, 31(1) : 46-54.

[4] HUANG Xiaotao,LIANG Diannong. Parametric methods ofRFI suppression in UWB-SAR[J].Journal of Systems Engineeringand Electronics， 2000，22( 2) : 94-97.

[5] WANG X Y, YU W D, QI X Y, et al.RFI suppression inSAR based on approximated spectral decomposition algorithm[J]. Electronics Letters, 2012, 48(10) : 594-596.

[6] BUCKREUSS S. Filtering interference from P-band SAR data [C] / / EUSAR98. [S. l.] , 1998: 279-282.

[7] ZHOU F,WU R, XING M, et al. Narrow band interferencesuppression for SAR using Eigen-subspace based filtering[J].IEEE Geoscience and Remote Sensing Letters, 2007, 4( 1) : 75-79.

[8] D. L. Donoho, “Compressed sensing,” IEEE Trans. Inf. Theory, vol.52, no. 4, pp. 1289-1306, 2006.

[9] Wax M, Kailath T. Detection of signals by information theoreticcriteria[J].IEEE Transactions on Speech and Signal Processing,1985,33 (2) : 387- 392. 